\title{
Vaginal cervicoisthmic cerclage for cervical incompetence in pregnant women: Fernandez's technique in 8 steps
}

\section{Margaux Jegaden', Elodie Debras ${ }^{1,2}$, Anne-Gaëlle Pourcelot ${ }^{1}$, Marie-Emmanuelle Neveu ${ }^{1}$, Perrine Capmas ${ }^{1-3}$ and Hervé Fernandez ${ }^{1-3 *}$}

${ }^{1}$ Department of Gynecology and Obstetrics, AP-HP, GHU-Sud, Hospital Bicêtre, 78 rue du Général Leclerc, 94270 Le Kremlin Bicêtre, France

2Université Paris-Saclay, UVSQ, Inserm, CESP, 94807, Villejuif, France

${ }^{3}$ Faculty of Medicine, University Paris-Sud Saclay, 63 rue Gabriel Péri, 94270 Le Kremlin, Bicêtre, France

\section{Abstract}

Video objective: To demonstrate that surgical technique of vaginal cervicoisthmic cerclage must be performed in women with history of cervical incompetence with more than two late miscarriages before 24 weeks or premature deliveries before 28 weeks and after prior failure of preventive Mc Donald cerclage. In this video, the authors describe the complete procedure in 8 steps to standardize and facilitate the procedure in a simple and safe way during pregnancy.

Design: Step-by-step video demonstration of the surgical technique.

Setting: Tertiary Center for University Hospital.

\section{Interventions}

Under spinal anaesthesia, the patient was placed in a modified lithotomy position. She received intravenous prophylactic antibiotic treatment (penicillin, unless contraindicated) during the procedure. The bladder was empty before the surgery.

Step 1 began with a cervical infiltration of lidocaïne 1\% diluted dose in serum saline.

Step 2 was anterior semicircular colpotomy on the cervicovaginal junction.

Step 3, the bladder was retracted until exposure of the cervicoisthmic junction and reclined with a vaginal valve.

Step 4 was posterior colpotomy with opening the recto uterine poach of Douglas and positioning of a posterior valve.

Steps 5 and 6 were bilateral insertion of a non-resorbable monofilament polypropylene sling (I-STOP ${ }^{\circledR}$, CL Medical Sainte Foy Les Lyon, France) around the cervicoisthmic junction

\section{More Information}

*Address for Correspondence: Hervé Fernandez, Department of Gynecology and Obstetrics, Hospital Bicêtre, 78 rue du Général Leclerc, 94270 Le Kremlin Bicêtre, Email: herve.fernandez@aphp.fr

Submitted: September 21, 2021 Approved: November 11, 2021 Published: November 12, 2021

How to cite this article: Jegaden M, Debras E, Pourcelot AG, Neveu ME, Capmas P, et al. Vaginal cervicoisthmic cerclage for cervical incompetence in pregnant women: Fernandez's technique in 8 steps. Arch Case Rep. 2021; 5 : 032-033.

\section{DOI: 10.29328/journal.acr.1001054}

Copyright License: ( 2021 Jegaden M, et al. This is an open access article distributed under the Creative Commons Attribution License, which permits unrestricted use, distribution, and reproduction in any medium, provided the original work is properly cited.

Keywords: Ambulatory care; Cervical incompe tence; Failure of Mac Donald cerclage; Surgical technique; Vaginal way

\section{Check for updates}

OPEN ACCESS above the uterosacral ligaments and below the arch level of the uterine artery. Anatomical landmarks are identified by visualization and digital palpation.

Step 7, after tightening, the tape was fixed to the anterior surface of the isthmus with two non-resorbable sutures on each arm of the sling.

Step 8, closure of the anterior and posterior colpotomy by a suture of resorbable thread.

The surgery was performed between 11 and 13 weeks of gestation within 30 minutes. Whoever the operators are, intraoperative complications are exceptional and only represented by the visibility to open the pouch of Douglas. In

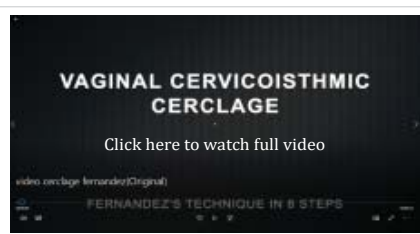

Video: Fernandez's technique in 8 steps. (https://youtu.be/zpCciYWzmqU) 
this case, the sling will be positioned lower in the posterior part of the cervix. Patient was discharged on the same day after confirming with ultrasound scan the embryonic viability and the position of the sling.

A cesarean delivery was planned around 37-38 weeks of gestation. It can be performed earlier in case of spontaneous labor or preterm membranes rupture with signs of infection.

This cerclage is permanent due to the high risk of bleeding during removal process, also because the sling is embedded in the myometrium.

\section{Conclusion}

Vaginal cervicoisthmic cerclage for cervical incompetence in pregnant women after previous failure of Mc Donald cerclage is safe and reproducible. This permanent cerclage does not need to be repeated for each subsequent pregnancy. Our team [1] report that neonatal survival rate after 24 weeks of gestation for the next pregnancy with the same cerclage was $96.3 \%$. We reported 1\% long-term complication of cervical erosion that needs to be removed. The complication rate is comparable to the one of sub-urethral tape.

\section{References}

1. Neveu ME, Fernandez H, Deffieux X, Senat MV, Houllier M, et al. Fertility and pregnancy outcomes after transvaginal cervico-isthmic cerclage. Eur J Obstet Gynecol Reprod Biol. 2017; 218: 21-26. PubMed: https://pubmed.ncbi.nlm.nih.gov/28926726/ 